\title{
A Feasibility Study of a Modified Treatment Strategy Combined External Beam Radiation Therapy and Brachytherapy for Cervical Cancer
}

\author{
Qi Fu \\ National Cancer Center, Chinese Academy of Medial Sciences and Peking Union Medical College \\ Wei Li \\ National Cancer Center, Chinese Academy of Medial Sciences and Peking Union Medical College \\ Jing Zuo ( $\sim$ zuojing01894@163.com) \\ National Cancer Center, Chinese Academy of Medial Sciences and Peking Union Medical College \\ Xi Yang \\ National Cancer Center, Chinese Academy of Medial Sciences and Peking Union Medical College \\ Yingjie Xu \\ National Cancer Center, Chinese Academy of Medial Sciences and Peking Union Medical College \\ Manni Huang \\ National Cancer Center, Chinese Academy of Medial Sciences and Peking Union Medical College \\ Jusheng An \\ National Cancer Center, Chinese Academy of Medial Sciences and Peking Union Medical College \\ Shuangzheng Jia \\ National Cancer Center, Chinese Academy of Medial Sciences and Peking Union Medical College \\ Lingying Wu \\ National Cancer Center, Chinese Academy of Medial Sciences and Peking Union Medical College
}

\section{Research Article}

Keywords: Cervical cancer, External beam radiation therapy, Brachytherapy, Volumetric-modulated arc therapy, Midline block, Central shielding

Posted Date: February 1st, 2022

DOI: https://doi.org/10.21203/rs.3.rs-1296042/v1

License: (1) This work is licensed under a Creative Commons Attribution 4.0 International License. Read Full License

Version of Record: A version of this preprint was published at Journal of Applied Clinical Medical Physics on April 21st, 2022. See the published version at https://doi.org/10.1002/acm2.13621. 


\section{Abstract}

Purpose: To evaluate the feasibility of a modified treatment strategy combined external beam radiation therapy (EBRT) and brachytherapy (BT) for cervical cancer through a dosimetry analysis.

Material and methods: This study retrospectively selected 12 cervical cancer patients treated with the conventional treatment strategy, which consisted of 45-50 Gy/25 fractions of EBRT using volumetric-modulated arc therapy (VMAT) and image-guided BT with fraction dose of 5-7 Gy. The modified treatment strategy decreased the central EBRT dose while increasing the BT fractions. New target volumes were additionally contoured and new VMAT EBRT plans were generated for the modified treatment strategy. The dosimetric parameters for evaluation included the doses to the most irradiated $2 \mathrm{cc}$ (D2cc) of the organs at risk (OARs) and the doses to at least 90\% (D90) of the gross tumor volume (GTV) and high-risk clinical target volume (HR-CTV). The total doses to OARs and targets that obtained by adding the equivalent doses in 2 Gy fraction (EQD2) from the EBRT and BT plans were used for quantitative comparison between the modified and conventional treatment strategies.

Results: Comparison to the conventional treatment strategy, the modified treatment strategy resulted in a higher bladder D2cc, a slightly lower rectal D2cc and a similar HR-CTV D90, all with no significant differences (pष0.05). The GTV D90 of the modified treatment strategy was significantly higher than that of the conventional treatment strategy ( $\mathrm{p} \otimes 0.01)$.

Conclusion: The modified treatment strategy can significantly increase the BT dose while remaining the total doses to the bladder and rectum basically unchanged, demonstrating its feasibility and promising prospect in clinical use.

\section{Introduction}

Radiation therapy is a main treatment for patients with locally advanced cervical cancer, which typically consists of external beam radiation therapy (EBRT) and brachytherapy (BT). The EBRT part of treatment aims to treat the whole pelvic, including the entire cervix, lymph nodes at risk and a part of bladder and rectum. Recommended EBRT dose is 45-50 Gy in 1.8-2.0 Gy/fraction. The BT part of treatment serves as a boost to EBRT, aiming to irradiate the residual tumor with multiple fractions. According to published guidelines [1], the total dose from EBRT and BT should reach at least 80-87 Gy (EQD2, expressed as equivalent dose in 2 Gy fraction) for effective local tumor control. Meanwhile, the minimum cumulative dose from EBRT and BT to the most irradiated 2 cc (D2cc) should not exceed 80-90 Gy (EQD2) and 65-75 Gy (EQD2) for bladder and rectum, respectively. Although BT is used as adjuvant therapy after EBRT, we typically want significant prescription dose could be delivered with BT because of its ability to provide high localized dose to tumor and extremely steep dose gradients to spare of normal tissues. However, the conventional EBRT treatment to the whole pelvis already results in high doses to bladder and rectum (even higher than prescription dose due to non-uniform dose distributions). In order to reduce radiological toxicity, the BT dose usually cannot be increased any more.

Aiming at this problem, many treatment centers used to place a midline block in external irradiation fields to reduce the EBRT dose in the midline region so that the significant dose could be delivered with BT [2-4]. However, this approach only applied to the EBRT using conformal radiation therapy (CRT). Nowadays, intensity-modulated radiation therapy (IMRT) and volumetric-modulated arc therapy (VMAT) have displaced CRT as common techniques for EBRT because of superior dose distributions and lower radiological toxicity [5-9]. To take the advantages of these advanced techniques, we developed a modified treatment strategy using VMAT to decrease the doses to bladder and rectum from EBRT while increasing the dose from BT by adding the BT fractions. This study presented this modified treatment strategy, evaluated its dosimetric parameters and compared it with the conventional treatment strategy.

\section{Materials And Methods}

Patient selection and contouring

This study included 12 cervical cancer patients treated with VMAT EBRT and more than 3 fractions of CT-guided high-dose-rate (HDR) BT using tandem/ovoid (T/O) applicators or T/O applicators with interstitial needles. According to FIGO stage classification [4], the local tumor stages of the patients were from Stage lb2 to IIIC2r. All patients underwent CT scans using a Brilliance CT Big Bore (Philips, Amsterdam, Netherlands) with 5-mm slice thickness for EBRT and 3-mm slice thickness for BT, respectively. The clinical target volume (CTV) of EBRT consists of the gross tumor volume (GTV), whole uterus and cervix, at least the upper half of the vagina, bilateral parametrium, uterosacral ligaments, and lymph nodal region at risk. A 5-mm expansion was applied to the CTV to create the planning target volume (PTV). Besides, our modified EBRT needs to additionally contour a PTV $\mathrm{U}_{\mathrm{U}+\mathrm{C}}$ and a PTV-PTV $\mathrm{V}_{\mathrm{U}+\mathrm{C}}$. The PTV Th+C $_{\mathrm{U}}$ consists of the uterus, cervix, and the top of the vagina, aiming to include most regions the BT dose could cover. The PTV-PTV $\mathrm{U}+\mathrm{C}$ is the remaining part of 
the PTV after subtracting the PTV $\mathrm{U}_{+\mathrm{C}}$ and adjacent bladder and rectum, as shown in Fig. 1. The target volumes of BT include GTV and high-risk CTV (HR-CTV), both contoured in accordance with GEC ESTRO recommendation [10]. Normal tissues including the bladder, rectum, sigmoid, colon, intestine, right and left femoral heads, pelvic bones were also contoured for planning and dose evaluation.

Prescriptions

The prescription doses (PD) of the conventional EBRT were 45-50 Gy to the PTV and a boost dose of 10-15 Gy to the enlarged lymph nodes in 25 fractions. To spare the bladder and rectum, we reduced the number of fractions from 25 to 21 and decreased the irradiation dose to the PTV $\mathrm{U}_{\mathrm{U}+\mathrm{C}}$. The prescription doses of the modified EBRT were $35.7 \mathrm{~Gy}$ to the PTV $\mathrm{U}_{\mathrm{C}+\mathrm{C}}$ and $45.15 \mathrm{~Gy}$ to the PTV-PTV $\mathrm{U}_{+\mathrm{C}}$. Enlarged lymph nodes still received extra boost dose of 10-15 Gy. For the BT part of treatment, the prescription dose to HR-CTV were 5-7 Gy/fraction. The difference between the modified BT and the conventional BT is the number of fractions. We increase the modified BT fractions from 4 to 6 . All the prescription doses were listed in Table 1.

Table 1 The prescription doses and the corresponding EQD2 of the modified and conventional treatment strategies.

\begin{tabular}{|llllll|}
\hline Treatment & Target & PD per fraction (Gy) & Fractions & PD (Gy) & EQD2 (Gy) \\
\cline { 2 - 6 } Modified EBRT & PTV $_{\mathrm{U}+\mathrm{C}}$ & 1.7 & 21 & 35.7 & 34.81 \\
\cline { 2 - 6 } & PTV-PTV $_{\mathrm{U}+\mathrm{C}}$ & 2.15 & 21 & 45.15 & 45.71 \\
\cline { 2 - 6 } & Lymph nodes & $2.6-2.8$ & 21 & $54.6-58.8$ & $57.33-62.72$ \\
\hline Modified BT & HR-CTV & $5-7$ & 6 & $30-42$ & $37.5-59.5$ \\
\hline Conventional EBRT & PTV & $1.8-2$ & 25 & $45-50$ & $44.25-50$ \\
\cline { 2 - 6 } & Lymph nodes & $2.2-2.4$ & 25 & $55-60$ & $55.92-62$ \\
\hline
\end{tabular}

Treatment planning

All EBRT VMAT plans were designed using Pinnacle v9.1-16.2 (Philips Radiation Oncology Systems, Fitchburg, WI, USA) and delivered using double full arcs with 6-MV X-ray. Dose constraints for normal tissues were as follows: rectum: maximum dose <52 Gy, volume receiving >50 Gy (V50) <20\%; sigmoid: maximum dose <52 Gy, V40<60\%; bladder: V50 <20\%; intestines: maximum dose <52 Gy, V40<50\%; spinal cord: maximum dose $<40 \mathrm{~Gy}$; femoral heads: V30<30\%, pelvic bone marrow: V30 <50\%; kidneys: V20<20\%. The plans were normalized to ensure that $95 \%$ of the target volumes received $100 \%$ of the prescription dose. The BT planning was performed using Oncentra Brachy v4.6 (Elekta Brachytherapy, Veneedal, The Netherlands), with a ${ }^{192}$ Ir source for a Flexitron afterloader unit. The activation step was $2 \mathrm{~mm}$. The source dwell times for each BT plan were optimized by hybrid inverse planning optimization (HIPO) algorithm and manually adjusted. The dose constraints used for optimization followed NCCN clinical practice guideline [5]. All the BT plans were normalized so that $90 \%$ of the HR-CTV (D90) received $100 \%$ of the prescribed fraction dose. Treatment planning in this study was done by a single experienced planner.

Dosimetric evaluation

The dosimetric parameters including D2cc of bladder and rectum and D90 of the GTV and HR-CTV were individually recorded for all plans. The boost doses to the parametrial lymph nodes will not contribute to the BT boost region but may increase the D2cc values. Thus, for both modified and conventional EBRT plans we only computed the D2cc of central bladder and rectum, which were the overlaps between these organs and the PTV $\mathrm{U}_{\mathrm{C}}$ expanded by $5 \mathrm{~cm}$ in anterior-posterior direction, as shown in Fig. 1. As the GTV and HR-CTV were fully contained in the PTV (or PTV $\mathrm{U}_{+\mathrm{C}}$ ), for simplicity, their D90 were considered to be the same as the prescription dose of the PTV (or PTV $\mathrm{U}_{+\mathrm{C}}$ ). For BT, suppose one plan could be implemented two times, then the dose volume parameters of each plan would be counted twice. The modified treatment needed to use three BT plans and the conventional treatment used first two BT plans, according to their corresponding numbers of fractions. The total doses for evaluation were the sum of doses from both EBRT and BT and normalized to EQD2 using the following formula:

$E Q D 2=n d \cdot \frac{d+\alpha / \beta}{2+\alpha / \beta}$ 
where $n$ was the number of fractions, $d$ was the fraction dose, $a$ and $\beta$ were the radiosensitivity parameters for the linear-quadratic responses, respectively. In this study, $\alpha / \beta$ for the targets and organs were 10 and 3 , respectively. The statistical significance of the dosimetric results was proven with a two-sided paired t-test at $5 \%$ level significance.

\section{Results}

Table 2 summarized the total D2cc of the bladder and rectum and the total D90 of the GTV and HR-CTV for the modified and conventional treatment strategies. The modified treatment strategy resulted in a slightly higher bladder D2cc ( $78.33 \pm 6.43$ vs $76.80 \pm 5.39)$, a slightly lower rectal D2cc ( $64.29 \pm 8.26$ vs $65.46 \pm 8.38)$ and a similar HR-CTV D90 (80.25 \pm 5.67 vs $79.77 \pm 4.18)$ in comparison to the conventional treatment strategy. But their values showed no significant differences $(p=0.167,0.405,0.595)$. Only the GTV D90 of the modified treatment strategy was significantly higher than that of the conventional treatment strategy ( $\mathrm{p} \otimes 0.01)$, which benefits from the increased fractions. Figure 2 shows an example of typical dose distribution for the modified EBRT plans.

Table 2 Comparison of total dosimetric parameters for the 12 patients between the modified and conventional treatment strategies (Gy).

\begin{tabular}{|c|c|c|c|c|c|c|c|c|}
\hline \multirow[t]{2}{*}{ Patient } & \multicolumn{2}{|c|}{ Bladder D2cc } & \multicolumn{2}{|c|}{ Rectum D2cc } & \multicolumn{2}{|l|}{ GTV D90 } & \multicolumn{2}{|l|}{ HR-CTV D 90} \\
\hline & Modified & Conventional & Modified & Conventional & Modified & Conventional & Modified & Conventiona \\
\hline 1 & 77.05 & 80.38 & 66.25 & 74.84 & 81.00 & 83.43 & 64.52 & 70.38 \\
\hline 2 & 77.66 & 74.86 & 71.53 & 73.63 & 107.16 & 98.70 & 82.81 & 82.00 \\
\hline 3 & 83.91 & 84.39 & 49.33 & 53.08 & 109.18 & 96.80 & 88.43 & 85.75 \\
\hline 4 & 66.49 & 70.66 & 69.19 & 72.51 & 109.63 & 99.99 & 86.64 & 85.83 \\
\hline 5 & 88.21 & 82.24 & 78.42 & 75.90 & 86.41 & 85.86 & 69.16 & 71.85 \\
\hline 6 & 83.60 & 79.86 & 70.09 & 70.52 & 104.46 & 95.14 & 82.84 & 82.04 \\
\hline 7 & 83.28 & 75.99 & 67.93 & 66.23 & 94.12 & 89.48 & 78.16 & 79.15 \\
\hline 8 & 80.27 & 81.87 & 55.29 & 51.70 & 94.39 & 89.63 & 77.52 & 78.50 \\
\hline 9 & 84.34 & 81.59 & 59.03 & 66.16 & 89.53 & 82.13 & 75.81 & 75.00 \\
\hline 10 & 72.83 & 69.04 & 52.33 & 54.05 & 126.12 & 112.10 & 86.64 & 85.83 \\
\hline 11 & 71.21 & 68.82 & 63.80 & 66.72 & 125.82 & 99.75 & 83.14 & 76.58 \\
\hline 12 & 71.12 & 71.86 & 68.34 & 60.15 & 101.31 & 92.99 & 79.31 & 76.25 \\
\hline Mean & $78.33 \pm 6.43$ & $76.80 \pm 5.39$ & $64.29 \pm 8.26$ & $65.46 \pm 8.38$ & $102.43 \pm 13.69$ & $93.83 \pm 8.10$ & $80.25 \pm 5.67$ & $79.77 \pm 4.18$ \\
\hline $\begin{array}{l}\text { p- } \\
\text { value }\end{array}$ & 0.167 & & 0.405 & & 0.002 & & 0.595 & \\
\hline
\end{tabular}

\section{Discussion}

Radiation therapy for cervical cancer is delivered by a combination of EBRT and BT. To fully take its advantage of high local tumor control and significant sparing of normal tissues, the BT dose should be increased as much as possible. Meanwhile, the doses to bladder and rectum from EBRT should be limited to reduce radiological toxicity. The common approach of achieving this is to use a midline block during EBRT to shield the central bladder and rectum. However, this approach has been in use for decades and unable to adapt to advanced techniques used in EBRT, such as IMRT and VMAT. Currently, there is very limited studies attempted to apply these advanced techniques to continues this approach. Dusten et al. developed a pseudo-split-field technique using IMRT based on the concept of midline block [11]. This technique was proved to be able to limit the central EBRT dose and maximize the BT dose. By adopting the centralshielding principle, Tomoaki et al. applied a 'two-step' VMAT in EBRT for early-age cervical cancer, which was likely to reduce radiation doses to rectum and small bowel [12]. In this study, we developed a modified treatment strategy combined VMAT EBRT and HDR BT. Compared to the 'two-step' VMAT mentioned above, our modified strategy employed a simpler 'one-step' VMAT with only 21 fractions and was easier to implement. From Figure 2, one can see that using VMAT technique well achieved the required EBRT dose distribution with many dose gradients and low dose in center. 
The goal of our strategy is to reduce the doses to bladder and rectum by decreasing the PTV $\mathrm{U}_{\mathrm{U}+\mathrm{C}}$ dose (from 45 Gy to $35.7 \mathrm{~Gy}$ ) and increase the BT dose by adding the BT fractions (from 4 to 6 ). The fraction dose to the PTV $\mathrm{U}_{+\mathrm{C}}$ for the modified EBRT is only 0.1 Gy lower than that for the conventional EBRT. This keeps the radiobiological effect of the modified EBRT on this region as closely as possible with that of the conventional EBRT. For the parametrial targets (PTV- PTV $\mathrm{U}_{+\mathrm{C}}$ ) and the involved lymph nodes, we increased their fraction doses so that their EQD2 were similar between the two EBRT. Results showed that our modified treatment strategy significantly increased the total D90 of the GTV while remaining the total D2cc of the bladder and rectum basically unchanged. This indicates that the modified strategy is likely to improve the local tumor control without an increase in the risk of radiological toxicity. Besides, the decrease in the central dose of EBRT may help to lower the incidence of radiation enteritis and colitis caused by positioning errors and changes in bladder filling. However, this is just a dosimetric study of this modified treatment strategy. The clinical usability and functional outcome of this strategy should be further investigated.

\section{Conclusion}

This study showed the dosimetric advantages of our modified treatment strategy for cervical cancer, demonstrating that it was feasible in clinical use. We therefore encourage other groups to test this strategy in clinical trials with us in future.

\section{Declarations}

\section{Ethics approval and consent to participate}

All procedures in this study were reviewed and approved by Cancer Hospital Chinese Academy of Medial Sciences.

\section{Consent for publication}

Publication of this study was approved by Cancer Hospital Chinese Academy of Medial Sciences.

\section{Availability of data and material}

The data that support the findings of this study are available from Cancer Hospital Chinese Academy of Medial Sciences but restrictions apply to the availability of these data, which were used under permission for the current study, and so are not publicly available. Data are however available from the authors upon reasonable request and with permission of Cancer Hospital Chinese Academy of Medial Sciences.

\section{Competing interests}

None.

\section{Funding}

This work was supported by the Beijing Hope Run Special Fund of Cancer Foundation of China (Grant No. LC2019B10).

\section{Authors' contributions}

Qi Fu, Wei Li, Jing Zuo, Xi Yang, Yingjie Xu, Manni Huang, Jusheng An, Shuangzheng Jia, Lingying Wu

Conception and design of the study - Qi Fu, Wei Li, Jing Zuo

Delineation and assessment of treatment - Wei Li, Xi Yang, Manni Huang, Jusheng An, Shuangzheng Jia Jusheng An, Manni Huang Acquisition and collection of data - Qi Fu, Wei Li

Analysis and interpretation of data - Qi Fu, Yingjie Xu, Jing Zuo, Xi Yang

Writing and revising the paper - Qi Fu, Yingjie Xu, Jing Zuo, Xi Yang

Final approval of the manuscript - Yingjie Xu, Jing Zuo, Lingying Wu

\section{Acknowledgements}


Not applicable.

\section{References}

1. Koh WJ, Abu-Rustum NR, Bean S, et al, Cervical cancer, version 3.2019, NCCN clinical practice guidelines in oncology. J Natl Compr Canc Netw. 2019;17:64-84. https://doi.org/10.6004/jnccn.2019.0001.

2. Wolfson AH, Abdel-Wahab M, Markoe AM et al. A quantitative assessment of standard vs. customized midline shield construction for invasive cervical carcinoma. Int J Radiat Oncol Biol Phys. 1997;37: 237-42. https://doi.org/ 10.1016/s0360-3016(96)00469-5.

3. Potter R, Knocke TH, Fellner C et al. Definitive radiotherapy based on HDR brachytherapy with iridium 192 in uterine cervix carcinoma: report on the Vienna University Hospital findings (1993-1997) compared to the preceding period in the context of ICRU 38 recommendations. Cancer Radiother. 2000;4: 159-72. https://doi.org/10.1016/S1278-3218(00)88900-3.

4. Huang EY, Lin $\mathrm{H}, \mathrm{Hsu} \mathrm{HC}$, et al. High external parametrial dose can increase the probability of radiation proctitis in patients with uterine cervix cancer. Gynecologic Oncology. 2000 Dec;79(3):406-410. https://doi.org/10.1006/gyno.2000.5997.

5. Mundt AJ, Lujan AE, Rotmensch $\mathrm{J}$ et al. Intensity-modulated whole pelvic radiotherapy in women with gynecologic malignancies. Int $\mathrm{J}$ Radiat Oncol Biol Phys. 2002;52:1330-7. https://doi.org/10.1016/s0360-3016(01)02785-7.

6. Hasselle MD, Rose BS, Kochanski JD et al. Clinical outcomes of intensity-modulated pelvic radiation therapy for carcinoma of the cervix. Int J Radiat Oncol Biol Phys. 2011;80:1436-45. https://doi.org/10.1016/j.ijrobp.2010.04.041

7. Chen MF, Tseng CJ, Tseng CC et al. Clinical outcome in posthysterectomy cervical cancer patients treated with concurrent Cisplatin and intensity-modulated pelvic radiotherapy: comparison with conventional radiotherapy. Int J Radiat Oncol Biol Phys. 2007;67:143844. https://doi.org/10.1016/j.ijrobp.2006.11.005

8. Sharfo AW, Voet PW, Breedveld S et al. Comparison of VMAT and IMRT strategies for cervical cancer patients using automated planning. Radiother Oncol. 2015;114:395-401. https://doi.org/10.1016/j.radonc.2015.02.006.

9. Marnitz S, Wlodarczyk W, Neumann O et al. Which technique for radiation is most beneficial for patients with locally advanced cervical cancer? Intensity modulated proton therapy versus intensity modulated photon treatment, helical tomotherapy and volumetric arc therapy for primary radiation-an intraindividual comparison. Radiat Oncol. 2015;10:91. https://doi.org/10.1186/s13014-015-0402-z.

10. Pötter R, Haie-Meder C, Limbergen EV, et al. Recommendations from gynaecological (GYN) GEC ESTRO working group (II): concepts and terms in 3D image-based treatment planning in cervix cancer brachytherapy-3D dose volume parameters and aspects of 3D image-based anatomy, radiation physics, radiobiology. Radiother Oncol. 2006 Jan;78(1): 67-77. https://doi.org/10.1016/j.radonc.2004.12.015.

11. Macdonald DM, Lin LL, Biehl K et al. Combined Intensity-Modulated Radiation Therapy and Brachytherapy in the Treatment of Cervical Cancer, International Journal of Radiation Oncology*Biology*Physics. 2008; 71(2): 618-624. https://doi.org/10.1016/j.ijrobp.2008.02.014.

12. Tamaki T, Hirai R, Igari M, et al. Dosimetric comparison of three-dimensional conformal radiotherapy versus volumetric-arc radiotherapy in cervical cancer treatment: applying the central-shielding principle to modern technology. J Radiat Res. 2018 Sep 1;59(5):639-648. https://doi.org/10.1093/jrr/rry054.

\section{Figures}




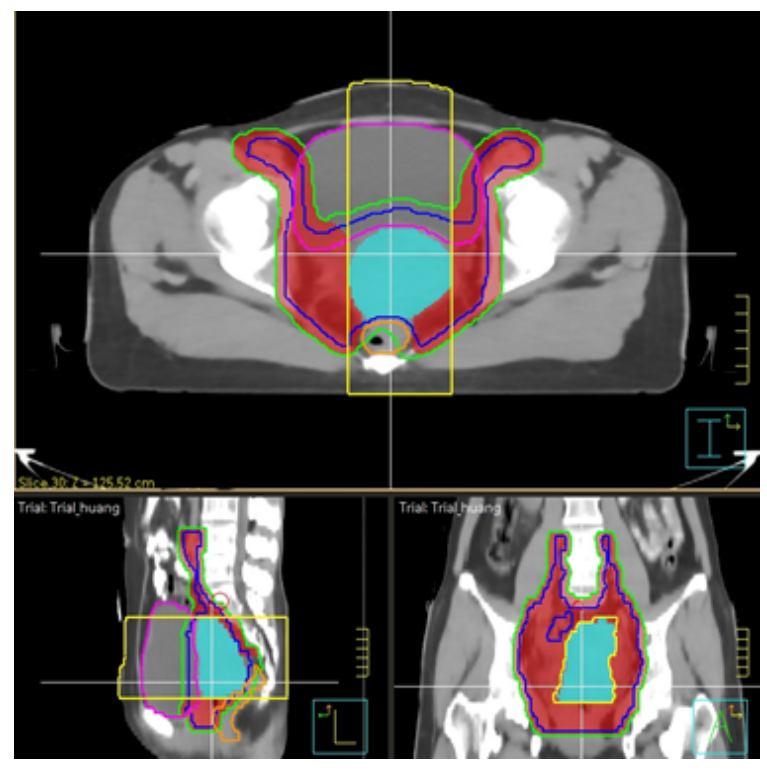

Figure 1

Axial, sagittal and coronal images showing the CTV (blue line), PTV (green line), PTV $\mathrm{U}+\mathrm{C}_{\text {(cyan area), PTV-PTV }}$ +C $($ red area), bladder (pink line), rectum (orange line), and the scope of D2cc evaluation (yellow line).

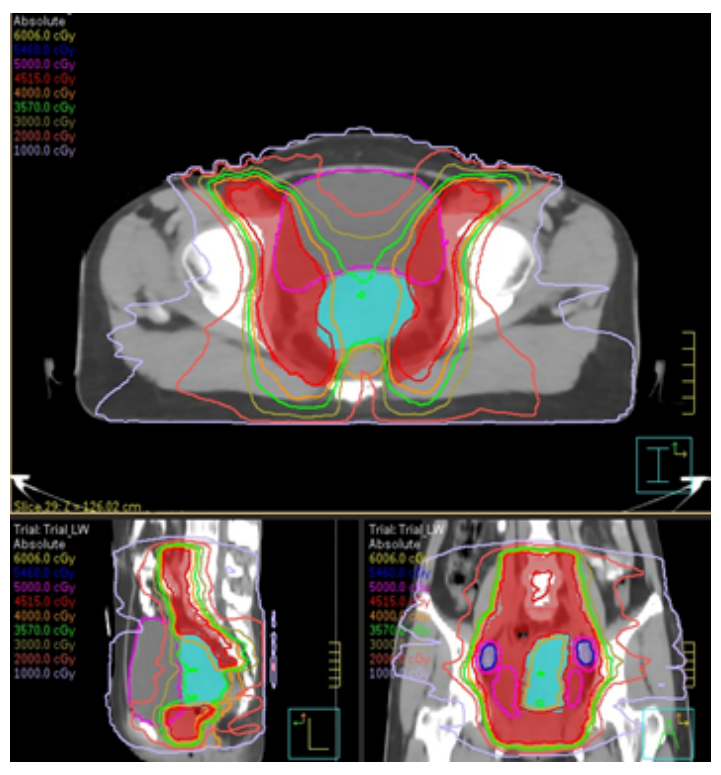

\section{Figure 2}

An example of dose distributions for the modified VMAT EBRT plans. 